Supporting Information

\title{
An engineered LPMO significantly boosting cellulase-catalyzed depolymerization of cellulose
}

Chao Cheng ${ }^{\mathrm{a}, \mathrm{b} \neq}$, Junaid Haider ${ }^{\mathrm{b}, \mathrm{c} \neq}$, Pi Liu ${ }^{\mathrm{b}, \mathrm{d}}$, Jianhua Yang ${ }^{\mathrm{b}, \mathrm{d}}$, Zijian Tan ${ }^{\mathrm{b}, \mathrm{d}}$, Tianchen Huang $^{\mathrm{b}, \mathrm{e}}$, Jianping Lin ${ }^{\mathrm{b}, \mathrm{d}}$, Min Jiang ${ }^{\mathrm{a} *}$, Haifeng Liu ${ }^{\mathrm{f} *}$, Leilei Zhu ${ }^{\mathrm{b}, \mathrm{d} *}$

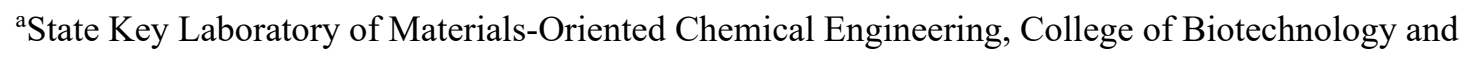
Pharmaceutical Engineering, Nanjing Tech University, Nanjing, 211800, P.R. China.

${ }^{b}$ Tianjin Institute of Industrial Biotechnology, Chinese Academy of Sciences, Tianjin 300308, P. R. China.

${ }^{c}$ University of Chinese Academy of Sciences, Beijing 100049, P.R. China.

${ }^{\mathrm{d}}$ National Technology Innovation Center of Synthetic Biology, Tianjin Institute of Industrial Biotechnology, Chinese Academy of Sciences, Tianjin 300308, P. R. China.

${ }^{\mathrm{e}}$ Department of Biological Engineering, College of Food and Bioengineering, Henan University of Science and Technology, Luoyang 471023, P. R. China.

fInstitute of Chemistry, University of Graz, NAWI Graz, BioTechMed Graz, Heinrichstrasse 28, Graz 8010, Austria.

¥These authors contributed equally

* Corresponding authors:

Prof. Dr. Leilei Zhu

E-mail: zhu_11@tib.cas.cn

Dr. Haifeng Liu

E-mail: haifeng.liu@uni-graz.at

Prof. Dr. Min Jiang

E-mail: bioengine@njtech.edu.cn

Number of pages: 9

Number of figures: 10

Number of tables: 1 


\section{Contents}

Table S1 Statistical analysis of hydrogen bonding between amino acid 458 and 526.

Fig. S1 Reaction mechanism of Amplex Red assay for LPMO activity determination. Fig. S2 Activity values in descending order of the HcLPMO10 WT using Amplex Red assay protocol in 96-well microtiter plate.

Fig. S3 Schematic illustration of screening strategy for improved variants.

Fig. S4 Relative activity of HcLPMO10 variants detected by Amplex Red assay.

Fig. S5 Linear calibration curves of cellulose depolymerization products, (a) gluconic acid and (b) glucose.

Fig. S6 Hydrogen peroxide production by HcLPMO10 (WT) cell lysate.

Fig. S7 Gluconic acid production from lactobionic acid (oxidized sugar) by $\beta$ glucosidase.

Fig. S8 The glucose production from HcLPMO10 in the presence and absence of cellulase.

Fig. S9 Conformation probability distribution of HcLPMO10WT and M1.

Fig. S10 RMSD (root mean square deviation) and RMSF (root mean square fluctuation) analysis of the MD simulation of HcLPMO10 WT and M1. 
Table S1. Statistical analysis of hydrogen bonding between amino acid 458 and 526.

\begin{tabular}{lcc}
\hline Hydrogen bond pairs & Frequency in WT & Frequency in M1 \\
\hline Glu458 (OE1,OE2)-526(side chain) ${ }^{\mathrm{a}}$ & 0.2085 & 0.2134 \\
Glu458 (O)-526(NH) & 0.0211 & 0.6767 \\
Total & 0.2296 & 0.8901 \\
\hline
\end{tabular}

${ }^{a}$ Note: In WT, the side chain atoms are Asn526 (ND2.HD21, ND2.HD22). In M1, the side chain atoms are Ser526 (OG.HG)

Optimization of fluorimetric screening system for LPMO in 96-well MTP format:

Two important parameters for a reliable MTP-based screening system are the true coefficient of variation (Fig. S2) and the linear detection range of the employed assay (Fig. S1b). The fluorimetric assay (Fig. S1a) was optimized for screening in 96-well MTP with a linear detection range of $\mathrm{H}_{2} \mathrm{O}_{2}$ (up to $5 \mu \mathrm{M}$ ). The true coefficient of variation was determined under optimal condition (25 $\mu \mathrm{M}$ Amplex Red, 7.5 U/mL HRP, $50 \mu \mathrm{L}$ cell lysate). After subtracting the background, the true coefficient of variation was $4.17 \%$ (Fig. S2). 
(a)

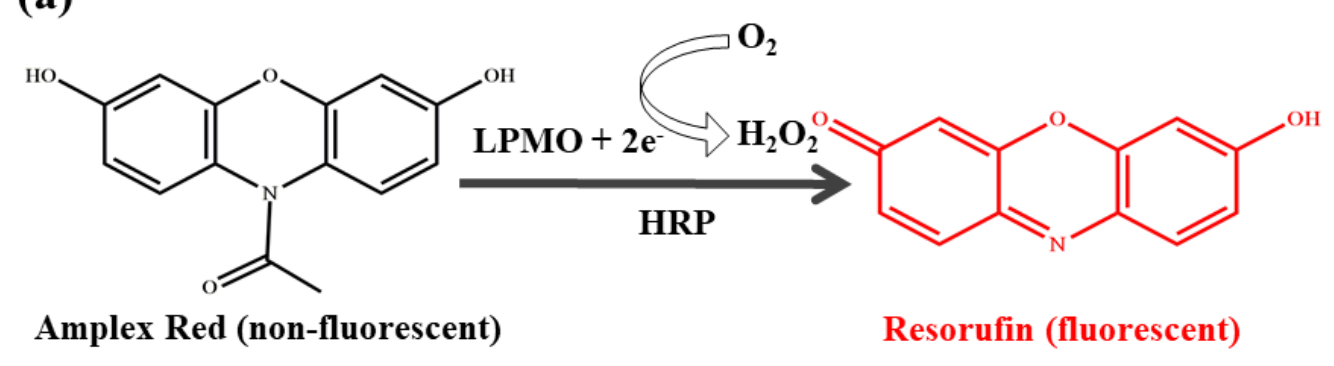

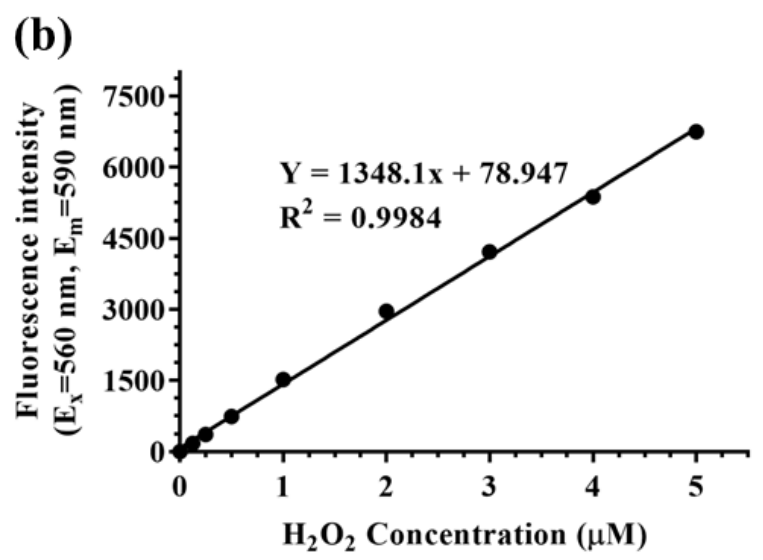

Fig. S1 Reaction mechanism of Amplex Red assay for LPMO activity determination. (a) LPMO produced $\mathrm{H}_{2} \mathrm{O}_{2}$ subsequently oxidizes Amplex Red to resorufin with fluorescent signal ( $\mathrm{E}_{\mathrm{x}}=560 \mathrm{~nm}$, $\mathrm{E}_{\mathrm{m}}=590 \mathrm{~nm}$ ) in the presence of HRP ${ }^{1}$. (b) Calibration curve of Amplex Red assay in 96-well MTP. Error bars represent the standard deviation of three independent experiments.

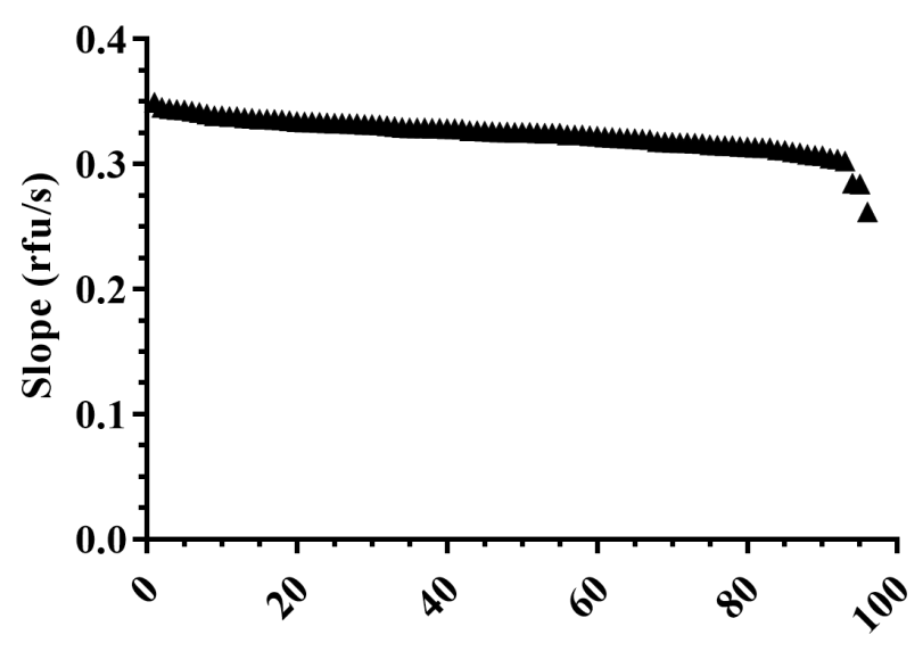

Clone number

Fig. S2 Activity values in descending order of the HcLPMO10 WT using Amplex Red assay protocol in 96-well microtiter plate. The true coefficient of variation was calculated after subtracting the background signal of reference samples without LPMO. 

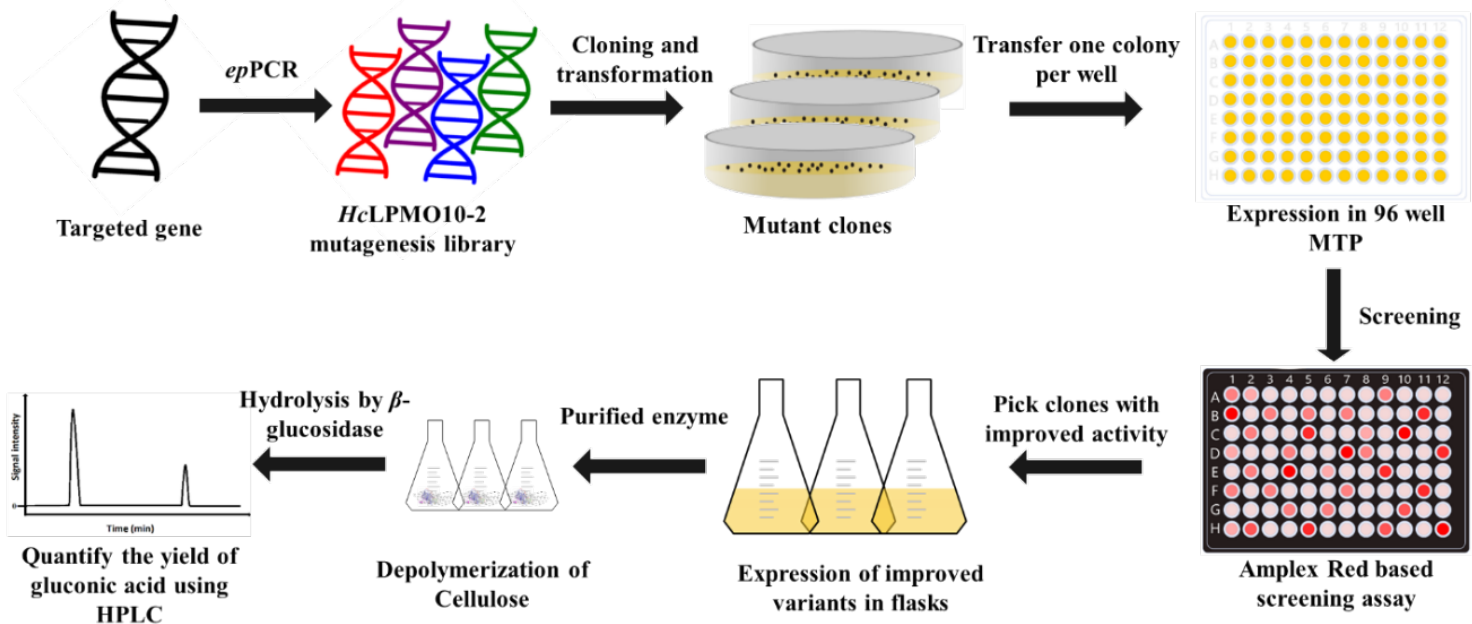

Fig. S3 Schematic illustration of screening strategy for improved variants. Gene diversity was generated via epPCR. Screening of mutant library is based on Amplex Red assay. For further identification, the improved variants were expressed in flasks and their cellulose depolymerization product, gluconic acid produced by hydrolysis of cello-oligosaccharide using $\beta$-glucosidase were quantified by HPLC.

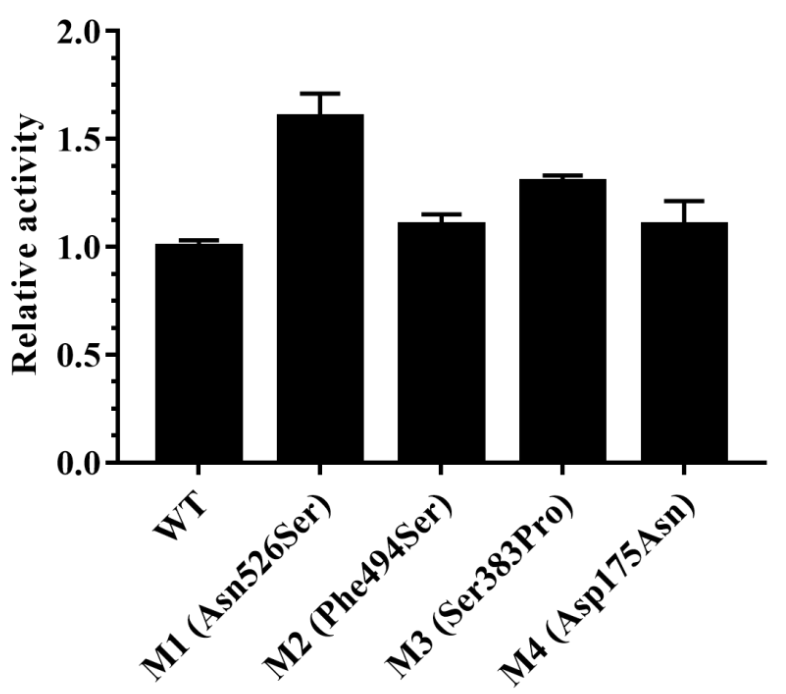

Fig. S4 Relative activity of $H c$ LPMO10 variants based on Amplex Red assay. The relative activity was defined as the activity of variant relative to the activity of $H c$ LPMO10 WT. The reaction mixture contained cell lysate $(50 \mu \mathrm{L})$, Amplex Red $(50 \mu \mathrm{M})$, HRP $(7.14 \mathrm{U} / \mathrm{mL})$, ascorbic acid (1 $\mathrm{mM}$ ), and $20 \mathrm{mM}$ sodium phosphate buffer ( $\mathrm{pH} 6.0$ ). All reactions were carried out at $30^{\circ} \mathrm{C}$ for $1 \mathrm{~h}$. 
(a)

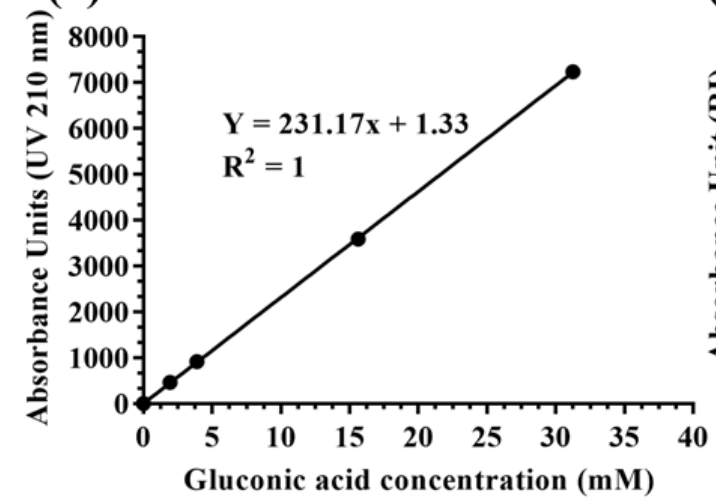

(b)

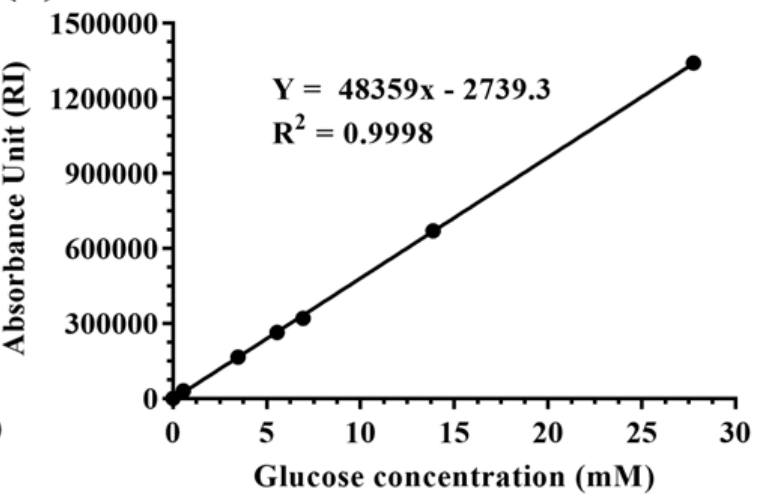

Fig. S5: Linear calibration curves of (a) gluconic acid and (b) glucose.

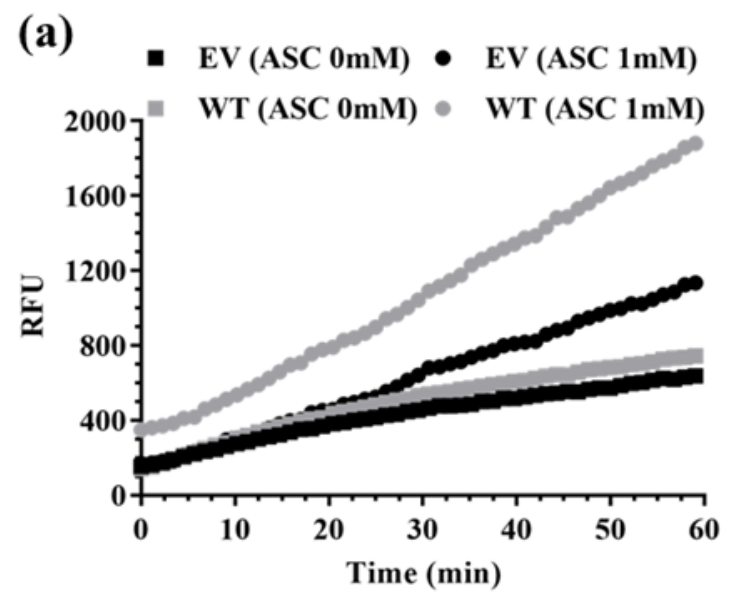

(b)

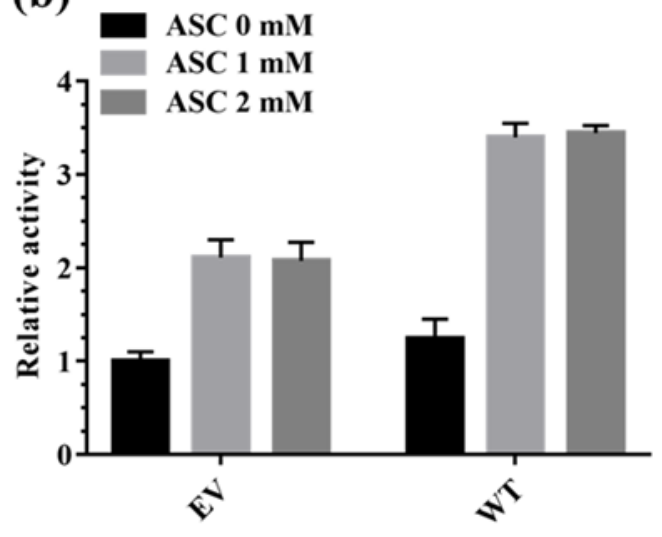

Fig. S6 Hydrogen peroxide production by HcLPMO10 WT cell lysate. (a) Fluorescence signals for measuring the production of $\mathrm{H}_{2} \mathrm{O}_{2}$ catalyzed by E.coli BL21 harboring empty vector (EV) and $H c$ LPMO10 (WT) cell lysate; (b) Relative activity of HcLPMO10 WT in the presence of various concentration of ascorbic acid ( $0-2 \mathrm{mM})$. The activity has been expressed as relative activity, i.e., $\mathrm{H}_{2} \mathrm{O}_{2}$ produced by WT relative to that of EV. Background signal of reference samples without LPMO were subtracted. All reactions were carried out in $20 \mathrm{mM}$ sodium phosphate buffer $\mathrm{pH} 6.0$ at $30^{\circ} \mathrm{C}$ for $1 \mathrm{~h}$. Error bars represent the standard deviation of the three independent experiments. 


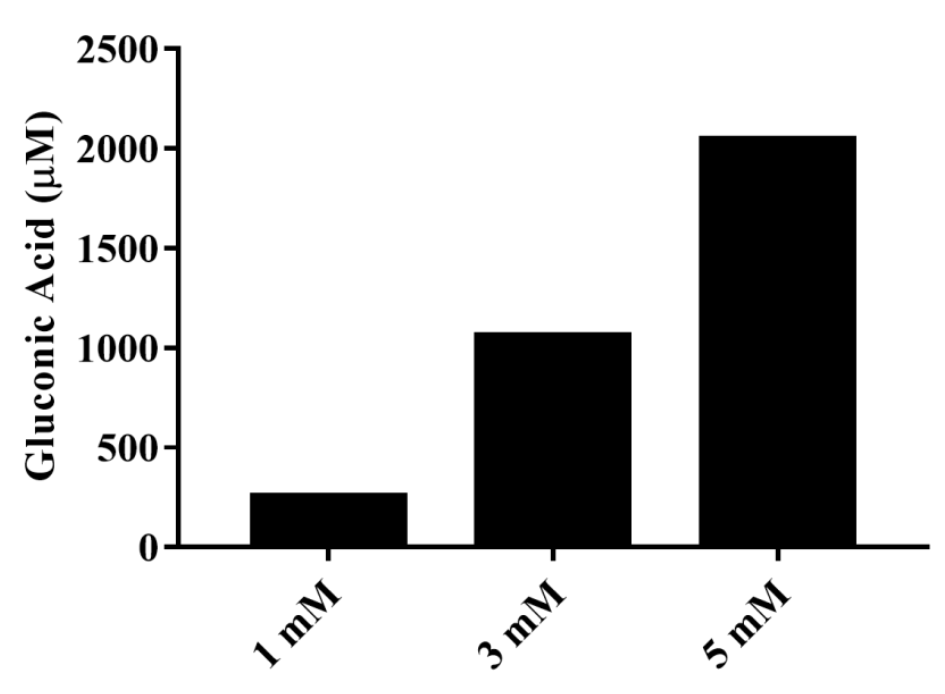

Fig. S7 Gluconic acid production from lactobionic acid (oxidized sugar) by $\beta$-glucosidase. Lactobionic acid (1-5 mM), and $\beta$-glucosidase $(10 \mathrm{U} / \mathrm{mL})$ were incubated in $50 \mathrm{mM}$ sodium phosphate buffer $\mathrm{pH} 6.0$ at $40^{\circ} \mathrm{C}$ for $24 \mathrm{~h}$.

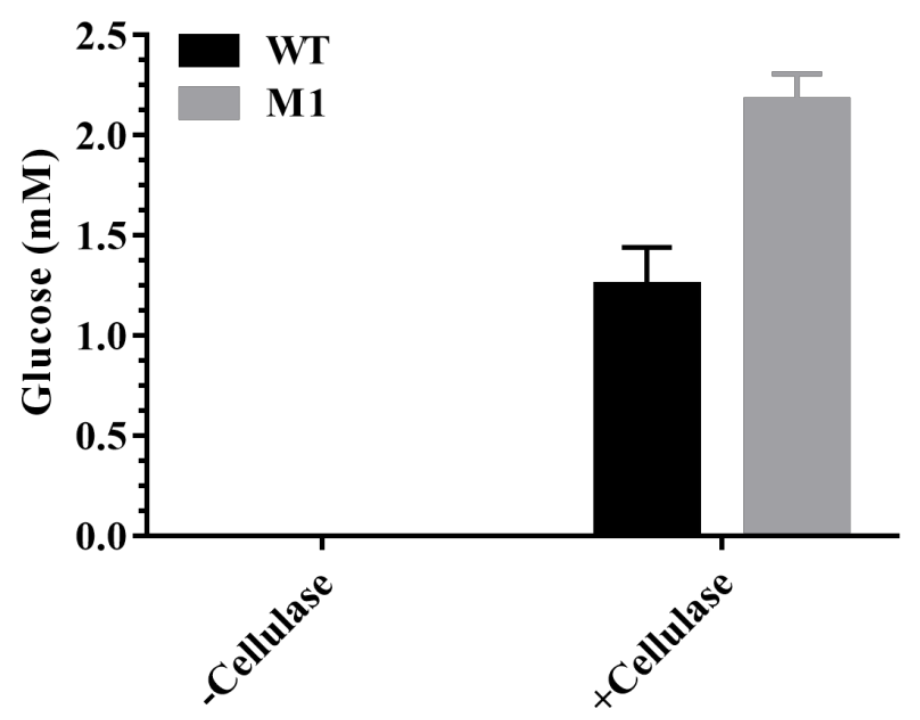

Fig. S8 The glucose production from $H c$ LPMO10 in the presence and absence of cellulase. Copper-saturated WT and M1 (2 $\mu \mathrm{M})$, Avicel (10 g/L), ascorbic acid (2 mM) and cellulase (3.12 $\mathrm{mg} / \mathrm{g}$ Avicel) were used in the experiment. Depolymerization of Avicel by $\mathrm{HcLMO} 10$ with and without cellulase was carried out in $20 \mathrm{mM}$ sodium phosphate buffer $\mathrm{pH} 6.0$ at $45^{\circ} \mathrm{C}$ for $48 \mathrm{~h}$. Error bars represent the standard deviation of the three independent experiments. 

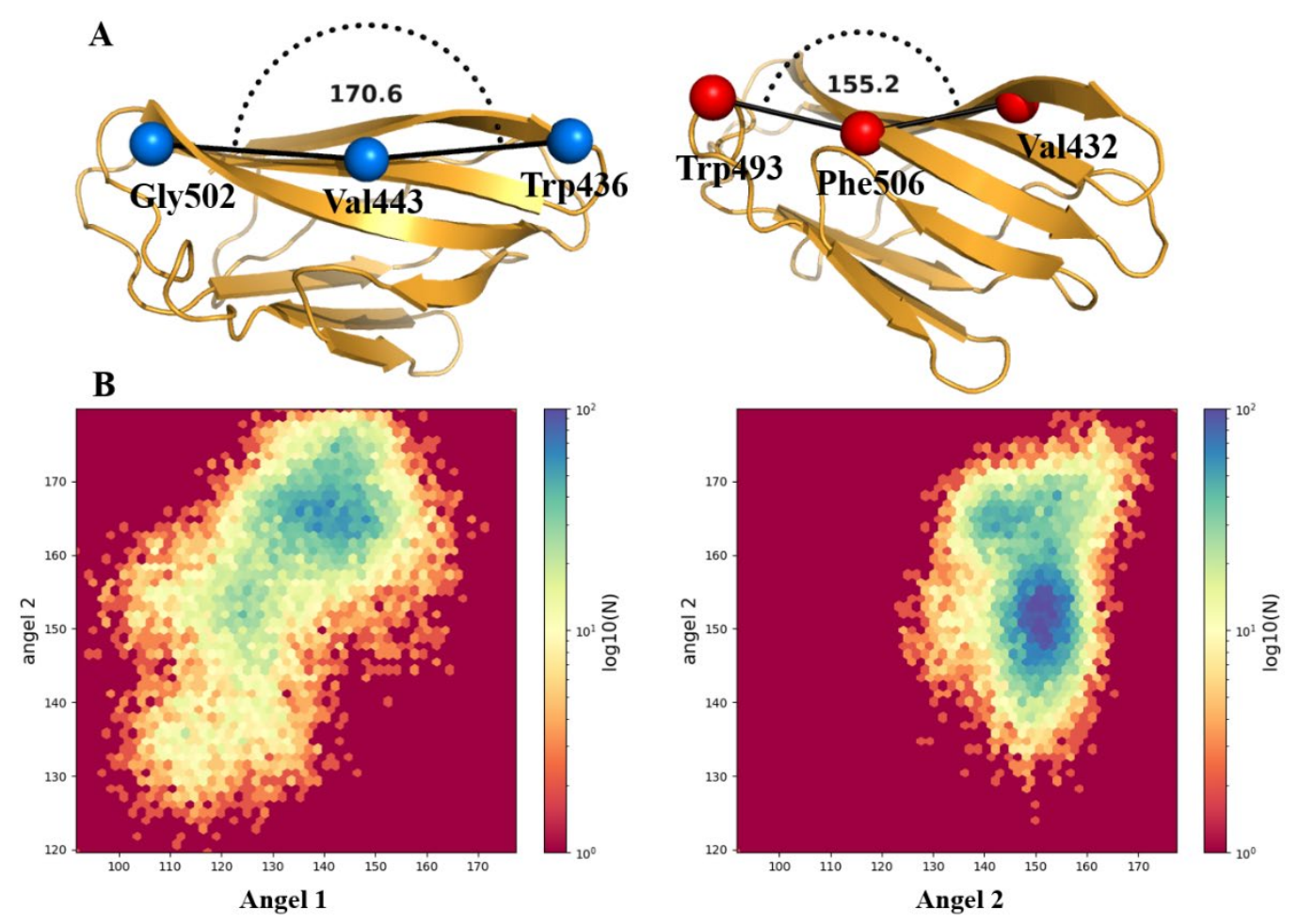

Fig. S9 Conformation probability distribution of $H c$ LPMO10 WT and M1. A: bending angle distribution and flattening angle. Angel 1 is defined by the angle between $\mathrm{C} \alpha$ atom of residue 436, 443 and 502; angel 2 is defined by the angle between $\mathrm{C} \alpha$ atom of residue 432, 506 and 493; B: conformation distribution probability map. 

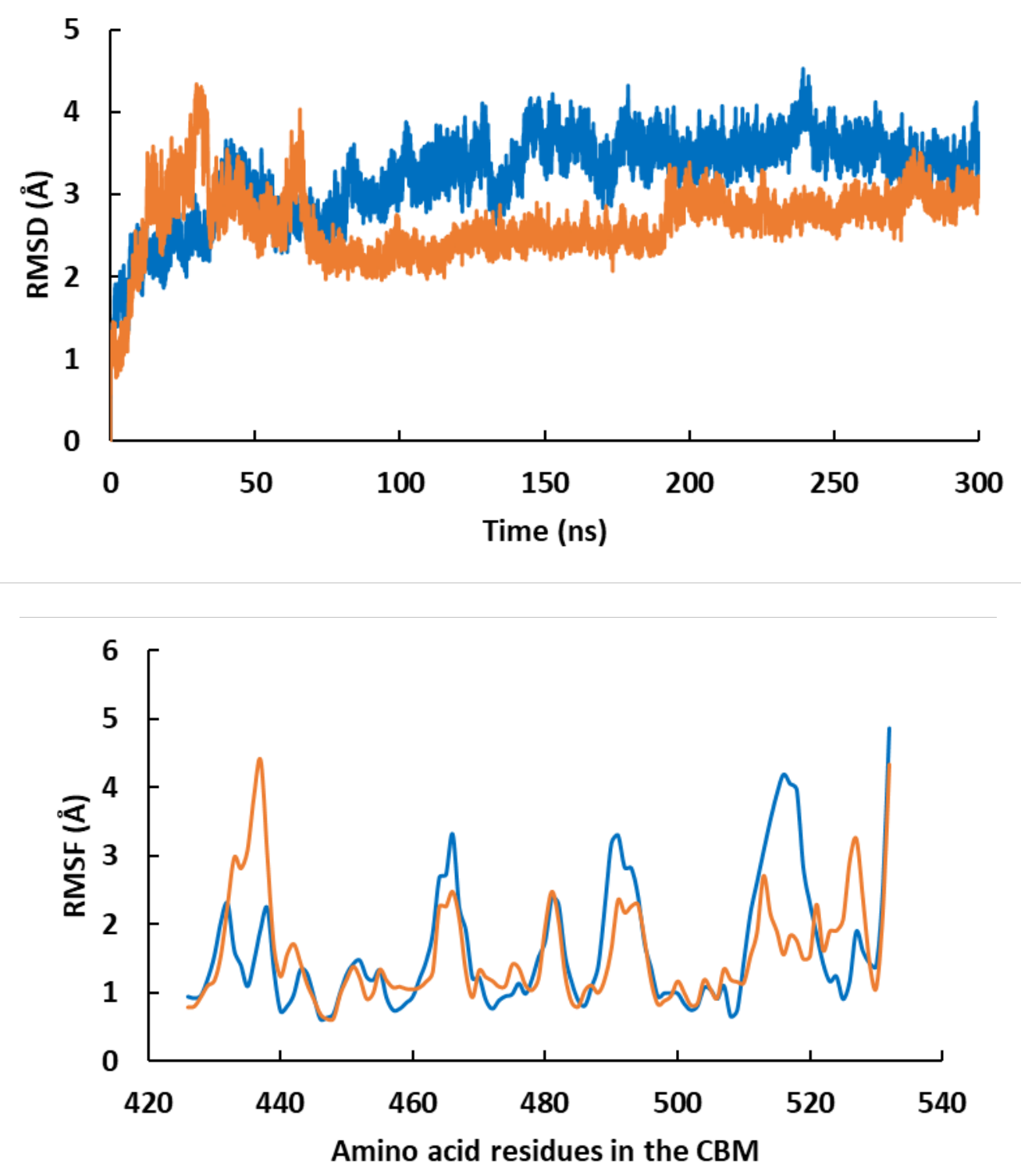

Fig. S10 RMSD (root mean square deviation) and RMSF (root mean square fluctuation) analysis of the MD simulation of $H c$ LPMO10 WT and M1.

\section{References:}

(1) Kittl, R.; Kracher, D.; Burgstaller, D.; Haltrich, D.; Ludwig, R. Production of Four Neurospora Crassa Lytic Polysaccharide Monooxygenases in Pichia Pastoris Monitored by a Fluorimetric Assay. Biotechnol. Biofuels 2012, 5 (1), 79. https://doi.org/10.1186/1754-6834-5-79. 